“Censorship", Early Childhood Research Quarterly and Qualitative Research: Not So Much Aced Out as an Own Goal? 


\begin{abstract}
As its starting point, this article investigates claims published in Qualitative Inquiry by Ceglowski, Bacigalupa, and Peck (2011) that Early Childhood Research Quarterly censored qualitative research. Unfortunately they assert rather than demonstrate political bias against qualitative research, fail to show that its publication in Early Childhood Research Quarterly has actually declined and ignore alternate hypotheses compatible with their data. After breaking their argument into parts, I find their censorship claims completely unsupported by evidence. However, this article has two larger aims. The first is to show how mistaking hypotheses for evidence, arguing unconvincingly from quantitative data, and failing to consider alternative interpretations of evidence weaken qualitative research, lowering its credibility within social science. The second is to consider the wider academic ramifications of publishing a peerreviewed journal article that totally fails to support its claims. Based on these concerns, the article offers some practical advice to avoid the negative outcomes demonstrated by the publication of Ceglowski, Bacigalupa, and Peck and considers the scientific implications of this rebuttal to their claims having been rejected previously by Qualitative Inquiry.
\end{abstract}

Keywords: qualitative research, censorship, interpretation, reliable inference, peer review 
“Censorship”, Early Childhood Research Quarterly and Qualitative Research: Not So Much Aced Out as an Own Goal?

In an article in Qualitative Inquiry (hereafter QI), Ceglowski, Bacigalupa, and Peck (2011, hereafter CBP) refer to three layers of censorship operating against qualitative research using, as an example, their experience of having a manuscript (hereafter ACS for “Allegedly Censored Submission”) rejected by Early Childhood Research Quarterly (hereafter ECRQ). CBP report that ACS was subsequently published elsewhere but it isn't possible to establish from CBP (or the web) where it was published (or under what title and authorship) so it cannot be cited directly. The use of all these acronyms is unfortunate but reflects the complexity of the example. In particular, it is necessary to avoid confusion when referring to the two distinct texts CBP and ACS.

In one sentence, the first element of my argument is that CBP do not provide any evidence that backs up the claim that ACS was censored by ECRQ. However, it is important (because I agree with CBP that qualitative research can be valuable and needs to be taken seriously) to consider three distinct ways in which they fail to do this (and what their wider implications are). The fact that CBP was considered suitable for publication in a peer-reviewed journal despite complete failure to support its claims constitutes prima facie evidence that the failings of this article may be symptomatic of more general weaknesses in qualitative research and its peer review process. To make this point as inescapably as possible, the arguments I use to undermine CBP's claims exclude value judgements about how social science should be done and mere appeals to authority by citation. This article shows the weaknesses of CBP directly from what they write (or fail to write) using only critical analysis. 
CBP's first failure is confusing hypotheses and evidence. This occurs in the first two sections of their article where the authors merely assert that qualitative research is not properly understood, that advocacy of randomised control trials (hereafter RCT) in educational research is politically (rather than scientifically) motivated and that presenting $\mathrm{RCT}$ as a research gold standard is merely a rhetorical ploy to privilege quantitative methods. The second failure is unconvincing use of quantitative methods to support their arguments about censorship. This occurs in the fourth section of CBP where the authors attempt to show that qualitative publication in ECRQ is declining as a supposed consequence of censorship. In fact, even the claim of declining publication is not supported as CBP present it (being sensitive to unjustified assumptions made in their analysis) and the alleged cause of this decline (censorship) is not demonstrated at all. The third failure is (ironically given CBP's stress on interpretation) not identifying (and adjudicating between) plausible alternative hypotheses compatible with the same data. This occurs in section 5 of CBP where a discussion of reviewer comments about ACS is presented as if it clearly shows evidence of misunderstanding (and perhaps partiality) when it can be interpreted equally plausibly to show normal reviewing practice, recognised differences in perspective about what constitutes good qualitative research, and concern for adequate rigour compatible with the practices of ECRQ.

The second (and potentially far more important) element of my argument is to consider the significance of the fact that an article entirely failing to support its claims has appeared in a peer-reviewed journal. What wider implication does this have for academic quality and what can be done about it? This issue is considered both in terms of the logic of peer review (and its inevitable fallibility) and through a reflexive analysis of my attempts to get the present article published and their implications. 
The article is organized as follows. The following section outlines the structure of the CBP article as background to detailed critique. The next three sections analyse in depth the three major failings identified in CBP: Mistaking hypotheses for evidence, arguing unconvincingly from quantitative data and failing to identify and adjudicate between different interpretations of the same evidence. The following section explains why CBP being published in a peer-reviewed journal creates a wider problem for the scientific process and why detailed critical rebuttals like the present article may thus be necessary. The next section considers suggestions for reducing the publication of qualitative research involving these failings in the context of a wider awareness of the scientific process as a whole. The final section examines the review process for the original version of this article sent to QI, its implications for the maintenance of qualitative research quality and the wider ramifications for scientific progress.

\section{The Structure of CBP}

CBP is organised into seven sections mapping reasonably straightforwardly onto the critique presented here. The first section (CBP, pp. 679-680) discusses the Reading Excellence Act (hereafter REA), asserting political motives for advocating RCT in education research and questioning whether critics and reviewers properly understand qualitative research. The second section (ibid., pp. 680-681) criticises the concept of "gold standards" in education research as an inappropriate rhetorical device that privileges quantitative research. The third section (ibid., pp. 681-682) discusses the scholarly significance of ECRQ as a leading journal in its field and presents data supposedly showing a decline in its publication of qualitative research after the REA. The fourth section (ibid, pp. 682-684) reports having ACS reviewed and rejected by ECRQ. The fifth section (ibid, p. 684) offers advice on successful submission of 
qualitative research. The sixth section (ibid., p. 685) reiterates earlier claims and the seventh section (ibid, p. 685) concludes. The critique in this article focuses mainly on sections two to five (the substantive parts of CBP) with sections two and three confusing hypotheses and evidence, section four using quantitative methods unconvincingly, and section five failing to identify and consider the significance of alternative interpretations of data. The next three sections of this article more thoroughly analyse the evidence for each failing in turn leading to the overall conclusion that no argument for the CBP claim of censorship withstands analysis.

\section{Hypotheses Are Not Evidence}

The first interesting thing about CBP is that the word censorship does not occur in the text anywhere but only in the title, abstract, and keywords. Censorship, one presumes, means rejecting research whose quality would normally justify publication for reasons other than quality. For example, CBP allege that ACS was rejected by ECRQ because it was qualitative, period. Clearly, non-publication results from at least two other common causes. (Although this article will not explore the point further, the three failures of CBP overlap repeatedly. Here, as later in their article, they attend to only one possible explanation without evidential justification.) The first is that a submission does not have the relevant quality required for the particular journal selected. The second is fallible peer reviewing. This involves mistakenly publishing articles the journal should not (given its quality and the quality of the submission) and rejecting acceptable ones. On reflection, such errors would hardly be surprising and can be observed empirically (Peters \& Ceci, 1982). CBP engage awkwardly with these possibilities. CBP imply that ACS was in fact good enough to be published by ECRQ (with the further implication that censorship is the reason ECRQ didn't publish it) 
because it was subsequently published (and in fact won a prize). However, it was published by a different journal about whose standards CBP tell us nothing. In fact, it does not seem possible to infer what that journal was from CBP and further web research also provides no likely citation. (As of 24 June 2013, Peck did not seem to have a homepage at all. Ceglowski had no CV or publications listed on hers and the online $\mathrm{CV}$ provided by Bacigalupa on her homepage listed neither an article by these three authors nor one that received a prize. The name of the prize and the university that awarded it were also not sufficient to identify the relevant article on the web. Dated PDF files for these searches are available from the author on request.) In these circumstances, the transparent thing for CBP to do would have been not only to make ACS available but also to cite its subsequently published incarnation. (After all, another possibility CBP don't discuss is that only improvements suggested by ECRQ reviewers rendered ACS publishable after all.)

Given the fallibility of reviewing, it is perfectly possible that the rejection by ECRQ correctly reflected the quality of ACS and that it was the acceptance by the other journal that was mistaken! In these circumstances, far from subsequent publication giving grounds for thinking there was anything wrong with the outcome of the ECRQ review process on ACS, the article could turn out to be poor even by the standards of the journal that did publish it! CBP cannot argue that an outcome is correct merely because it happens to suit them and problematic because it does not. In fact, of course, journals differ considerably in the proportion of submitted articles they reject and the average number of times the articles they do publish are cited. While caution must be used in interpreting these simplistically as measures of a quality - articles may be extensively cited for other reasons with the role of Mohammed El Naschie in the 
dramatic league table rise of the University of Alexandria as an interesting case (http://www.nytimes.com/2010/11/15/education/15iht-educLede15.html) - they should broadly tell us something about the ranking of journals. A quantitative researcher might point out that a sample larger than one case is necessary to draw any conclusions about reviewing outcomes when reviewers are fallible. In fact, systematic empirical research on peer review effectiveness exists - see Cole, Cole, \& Simon, 1981 and Weller, 2002 for overviews - but CBP do not cite it.

The correlation between quality and receiving prizes (the other part of the CBP justification for questioning the rejection of ACS by ECRQ) is not discussed and to my knowledge has not been researched. CBP only imply that a prize-winning article must be good though it does not follow self-evidently that it is good enough for ECRQ. As CBP point out on page 681, ECRQ, at the time, had an impact factor of 1.39 placing it $16^{\text {th }}$ highest in its category according to Thompson Reuters and making it a "top-tier journal”. By contrast, the prize - which web search cannot conclusively identify from CBP's description - appears to be a single university award. We thus have no way of telling how many individuals (and at what level of experience) were actually eligible for it and thus how strong the competition is in any particular year. It seems perfectly possible that even a prize-winning article at the university level might still not be good enough for a top-tier journal. The non-conspiracy hypothesis (that ACS was simply not good enough for ECRQ but was good enough for the other - presumably lower-quality - journal where it was published - and thus that peer review did what it is supposed to do) still stands and is more probable given the evidence (or lack thereof) that CBP present. 
Having shown that CBP offer no direct evidence for censorship, this section reaches its crux by demonstrating how mere hypotheses are offered instead. This happens in two distinct ways. On pages 679-680, CBP cite others who have also claimed that research standards may be politically motivated or otherwise nonscientific, but at no point do they report any evidence for these claims through discussion of the articles that they cite. In fact, even CBP's attempt to imply consensus is problematic. For example, it seems to me (though readers will have to decide for themselves) that the article by Simons (1995) which CBP claim "reports similar changes [in types of research]" (CBP, p. 680) is not making a similar argument to CBP even according to CBP's own presentation of what Simons says. Whether scientists should undertake government research whose results may be suppressed or appropriated (as CBP present the Simons argument) seems rather distinct from whether research is being censored by academic journals. The relevance of such distinctions vanishes only under such broad categories (like interference with the scientific process) that they are probably unusable. (Serial murder and littering can both be defined as crimes but what can usefully be said about the causes and prevention of crime so broadly defined?) The impression given is that CBP believe that if one cites enough people who share the same opinions then these become facts. What they actually become is factoids and that is why mere citation does not ensure scholarly quality. What is cited (arguments and evidence - whether qualitative or quantitative - not mere opinions) also matters. It should be noted in passing that CBP also cite loaded language ("so called evidence based", p. 679, "nakedly political”, p. 680) and potentially derogatory rhetoric ("aligned with positivism", p. 680) without justification or reservation. 
The second manifestation of hypotheses substituted for evidence is the section entitled "Master Narratives in Educational Research" (pp. 680-681). As much as anything, it is the willingness of CBP to present material like this section as an argument (and the willingness of a peer-reviewed journal to publish it) that does most harm to the credibility of qualitative research. It is, to be blunt, pure speculation. CBP describe a sequence of social processes by which the views of "a limited number of researchers - mostly white and male" (CBP, p. 680) may determine the legitimacy of different forms of research. For example, these individuals often control funding and can thus support or discourage different kinds of research and this, in turn, may socialise pragmatic junior staff into carrying out (and perhaps even promoting) research of kinds they approve. These are not implausible processes but there is not an argument or even a hint suggesting why we should believe this conspiracy account rather than other equally plausible and coherent narratives (for example that social science is a systematic but fallible search for truth). Including this section in the article presumably implies that CBP believe that hypothesizing a Master Narrative must have some bearing on their argument that ECRQ censored ACS but it is not clear to this reader (and CBP do not say) what that bearing is. One can only conclude that the reviewers and editors at QI found this hypothesis about Master Narratives sufficiently appealing that they didn't wish to oblige CBP to present evidence supporting it.

\section{Unconvincing Use of Quantitative Evidence}

A significant part of CBP's argument (pp. 681-682) supporting the claim that ACS was censored is the supposedly changing amount of qualitative research in ECRQ. This aspect of their argument is flawed on two levels. Firstly, it involves special pleading. CBP argue that one reason their article was rejected by ECRQ was that it was 
not properly reviewed (i.e. by qualitative researchers who agree with them about what qualitative research is, pp. 682-683). In that case, for consistency, a quantitative researcher should have reviewed at least this part of the QI article. (Of course, one cannot know if this actually occurred because reviewing is anonymous but it would be very surprising if a competent statistician had approved the conclusions from this section of CBP in the form published by QI. It would be easy to test this hypothesis by having sections of the published article peer reviewed by statisticians as if they were from a manuscript.) This point would not matter if their quantitative analysis led to justified conclusions but the second issue is that it does not. Specifically, CBP's arguments seem to rest on unjustified (and in some cases perhaps surprising) assumptions about how their data should be analysed which I shall discuss in detail shortly. It is unfortunate for CBP to claim that only initiates can understand qualitative research but to imply that anyone can use statistics and then do so in such an unconvincing way.

I shall discuss the CBP quantitative analysis in three parts in order to conclusively demonstrate its failure to support their claim of censorship. Firstly, I shall look at the way in which they allocated articles in ECRQ to categories such as qualitative and quantitative and the issues that arose when I tried to reconstruct this allocation. Secondly, I will examine the way in which they analysed the numbers in each category to reach their conclusions and show how their result (of declining qualitative publication in ECRQ) is sensitive to assumptions that CBP fail to justify (or even discuss). Finally, I will consider the implications for claims of censorship if one analyzes the distribution of qualitative and quantitative articles over the whole history 
of the journal (rather than two relatively short, arbitrary, and uneven periods as CBP do).

For an article advocating the value of qualitative research, CBP's discussion of their allocation of articles to qualitative and quantitative categories (p. 681) is surprisingly short and lacking in detail. To follow a very useful discussion by Brantlinger, Jiminez, Klinger, Pugach, \& Richardson, 2005, Figure 2, p. 201) discussing how qualitative research should establish its credibility, I can find no evidence in CBP of member checks (in which ECRQ might have been asked for a direct response to censorship allegations) or research reflexivity (in explaining clearly how and why articles in ECRQ were assigned to qualitative and quantitative categories for example). CBP report using a search engine on terms they thought suitable to identify qualitative research like $<$ case study $>$. However, without further explanation, they next give totals for qualitative and quantitative articles in ECRQ over two time periods before and after the REA (CBP, pp. 681-682). They also remark (about the search term <observations>), that "not all of these studies were qualitative" (CBP, p. 681). This suggests that the search was only used to identify articles and that there was some sort of actual content analysis too. But about that, absolutely nothing is reported except the resulting category totals. From the published CBP description, one cannot disallow the possibility that an article containing the phrase "ethnography is nonsense" would be found by the search engine and categorised as qualitative by CBP! Under these circumstances, it was impossible to follow CBP's procedure (and thus replicate their results) directly, but since the issues involved in deciding whether or not an article is qualitative can be defined reasonably clearly it seemed appropriate to try to understand the CBP results using a common sense (and, much more importantly, clearly specified) procedure for 
allocating articles to different categories by the research methods they use. This was to skim read each article in ECRQ, looking for respondent (rather than academic) quotations and numbers. If the meanings of the quotations were discussed, the results organised into themes and so on then the article was categorised as qualitative. If the data and analysis presented were numerical (regression, ANOVA and so on), then the article was categorised as quantitative. Obviously, if data of both kinds was presented, then my procedure allocated the article to the mixed category (only $4 \%$ of articles fell into this class for reasons discussed below) while if data of neither kind was present the article was placed in the no data category. (About $11 \%$ of articles were of this kind, being literature reviews or policy discussions for example.) Given that they do not discuss their procedures, it is impossible to say whether the analysis presented here should be more meaningful than that done by $\mathrm{CBP}$ but the reader can be sure (as they cannot from CBP's description) that my allocation procedure definitely involved reading (albeit rapidly) the whole of every article. (The data analysis spreadsheets are available on request from the author.) Thus in terms of the Brantlinger et al. (2005, Figure 2, p. 201) categories introduced above, I have made my case by drawing attention to other kinds of data (for example quantitative studies of peer review effectiveness), reflexively discussed a range of hypotheses and given arguments and evidence for those I prefer and subjected the results to peer review by both QI and ECRQ (member checks and/or external auditing).

Interestingly, the key issue affecting this part of CBP's argument (even though it is quantitative) is again one of interpretation, and it is rather surprising that the authors and QI's reviewers and editor did not seem to notice it. In the process of reconstructing CBP's analysis, it became clear that one had to be quite thoughtful in dividing ECRQ 
articles into qualitative and quantitative categories and at the very least show ones working (as CBP do not do). This kind of categorisation, intuitive though it seems, involves interpretive ambiguities that (given the small numbers on which CBP's argument appear to hinge) may have a significant impact on their claims. As an example of such an interpretive issue requiring a decision that may be necessarily arbitrary, should an article citing only descriptive statistics (such as averages) be considered mixed when the bulk of it is based on a narrative analysis of respondent quotations? (Such articles contain numerical data but no numerical analysis.) Similarly, should we consider an article collecting qualitative data (observations) but coding these quantitatively and doing statistical analysis on the results as qualitative, quantitative, or mixed? (Here there are narrative data but no narrative analysis. The relevance of the fact that there are many such articles in ECRQ will be discussed below.) The answer "no" seemed appropriate to both questions (and the reconstruction reported here took place accordingly). It also seemed appropriate not to allocate articles with a few anecdotal quotations to the mixed category. (It was these decisions that resulted in the fairly small proportion of mixed-methods articles. To qualify, an article had to report both qualitative and quantitative analysis not just data.) Finally, should articles with an experimental or evaluation design (including RCT) be treated as a separate category? In the results reported here, it seemed reasonable not to do this since these articles could still be allocated effectively into qualitative and quantitative categories according to my procedure (and it is the qualitative/quantitative distinction on which CBP's argument hinges after all). However, taken as a whole, the reconstruction process reported here made me aware of just how many issues of interpretation and procedure CBP had failed to make explicit in citing differences in (rather small) numbers of qualitative articles 
over two time periods as if categorisation was unproblematic. In particular, the number of ECRQ articles in which qualitative data were analysed quantitatively easily outweighs the number of articles that are traditionally qualitative. Though it is impossible to be sure, it seems CBP also allocated such articles to the quantitative category. However, given the ECRQ reviewer comments on ACS discussed by CBP, it is quite possible that some researchers would make a different (but equally reasonable) decision and, given the numbers involved, that this would have a major effect on the observed trend of qualitative publication in ECRQ. There may be no correct decision on this matter but it is hard to see how CBP briefly explaining only a few elements of what they did (and more importantly why they did it) can serve the interests of scientific knowledge.

For reference, CBP report (pp. 681-682) 12 qualitative articles between 1996 and 1998 and 4 qualitative articles between 2000 and 2003. My independently reconstructed allocation - not attending to the CBP totals until I had carried out my own procedure - gives 11 and 6 qualitative articles respectively. Thus the two allocation processes would seem not to be violently incompatible (though the very small numbers involved mean that this is about the most that can be said.) It is important to be clear about the implications of the discussion presented here. The argument is not that my analysis is right and that of CBP wrong (though mine is justified and explained while theirs is not). Instead, my argument is that the absence of context in CBP's allocation of articles to categories conceals too much that qualitative researchers would be concerned about in reaching a justified conclusion and that this absence of context had real bearing on their results and how they can plausibly be interpreted. As I shall now show, CBP's use of this categorisation would raise concerns with quantitative researchers too. 
Reconstructing the CBP allocation of articles by research method also makes it clear that even basic quantitative analysis must be presented with caution. This article begins by analysing all completed years of ECRQ at the time of writing (1986-2011) rather than the two fairly short segments either side of the REA (1996-1998 and 20002003) analysed by CBP. Dividing this sample into pre and post 1999 sections (thus following CBP in excluding the REA year of 1999), we find that the journal has grown only slightly (from an average of 7.2 to 7.9 articles per issue). Pre $1999,45 \%$ of issues contained a single qualitative article while post 1999 the figure was $24 \%$. In fact, the modal state for an issue became "no qualitative articles" post 1999 (and was only "one qualitative article" pre 1999). The number of issues with large numbers of qualitative articles relative to the mode (2+) also dropped considerably: From 9 before the REA in 1999 to 3 after. (Given the numbers involved, a single issue of ECRQ with 3 qualitative articles carries as much weight in analysis as the rest of the issues in a typical publishing year put together. I shall return to the implications of this point.) Under these circumstances, journal growth alone is insufficient to imply that the modal issue should now involve (for example) two qualitative articles. However, it probably justifies (as CBP do not) reporting articles using different research methods as fractions of a total rather than as raw counts. For example, had the journal started to publish significantly fewer (but longer) articles, the change in the raw number of qualitative articles might be explained by this alone.

The first thing to note about the numbers that $\mathrm{CBP}$ report is that the time periods analyzed pre and post REA (1996-1998 and 2000-2003) are different lengths (3 and 4 years) but no reason is given for this. As it happens, looking at time periods of equal size (either shortening the post REA period of analysis or lengthening the pre REA 
period) and looking at longer time periods either side of the REA (for example, six year periods: 1993-1998 and 2000-2005) do not change the basic conclusion that less qualitative research was published after the REA than before. However, much more damagingly, $\mathrm{CBP}$ also fail to remark on the significant role that the timing and content of special issues have on their results.

Figure 1 shows three kinds of data. The first column is the average fraction of the four different kinds of article (qualitative, quantitative, mixed, and no data) for all non-special issues of the journal. The next nine columns show the fractions for all the special issues published in ECRQ between 1986 and 2011. The final three columns show the fractions for three non-special issues of the journal selected randomly. It should be fairly clear both that the randomly selected issues show broadly similar distributions to the average fractions (such that this average is a useful summary of the non-special issues) and that most of the special issues are importantly different from this average in one or more ways (such that the average is not a useful summary for the nature of special issues). For example, one special issue consists entirely of non-data articles while another has far more qualitative research than average and so on. At best, only one special issue (or charitably two) involve distributions that look anything like typical non-special issues. Finally, it should be noted that the distribution of special issues by year is fairly uneven (17 years have no special issue, 6 have 1 and 2 have 2) and that only one of the nine special issues happens to occur after the REA.

The implications of these points (which CBP do not raise) are that comparisons over short time periods may be very sensitive to the presence and style of special issues. This is an argument for excluding special issues from the general characterisation of ECRQ regarding the research methods it publishes. Given the untypical nature of 
special issues (in content, timing and frequency), all it takes is one very quantitative special issue before the REA or one very qualitative one afterwards to shift the whole conclusion. Indeed, this is what we find. Using the same time periods as CBP but excluding special issues as untypical, one ends up with 5 qualitative articles in the first period and 5 after! In other words, the contingent timing and nature of special issues explains the whole difference between the before and after figures at least for the time periods that CBP chose to analyse. Again, my point is not that I am right and CBP wrong (I cannot judge if readers would agree, based on my argument, that it was reasonable to exclude special issues) but that if assumptions like this make so much difference to the results, then they need to be discussed and justified before the data presented can be considered to support the conclusions. CBP do not do this.

Interestingly, taking the lifetime of the journal as a whole (until 2011), CBP are actually correct that the publication of qualitative research in ECRQ is declining (although they fail to show it convincingly using their analysis). Here again, however, their decision to look only at short time periods is not justified and has very different implications for their allegations of censorship in connection with the REA. In this case, what makes their analysis unconvincing is that they fail to justify the link between the observed trend and any particular cause (like the operation of censorship).

Figure 2 shows the proportion of qualitative articles in ECRQ between 1986 and 2011 (as a fraction only of the population of qualitative and quantitative articles, thus excluding mixed methods and "no data" articles from the analysis. Special issues are also excluded for reasons discussed previously.) From this figure, it is possible to draw three relevant conclusions. Firstly, it is very clear that ECRQ is (and always has been) a strongly quantitative journal in which there was frequently little or no qualitative 
research in a given issue even long before the REA. The scale of change shown is only in fractions of an article (really in shifting likelihoods that any issue would contain a particular small number of qualitative articles) rather than in overall journal emphasis. Secondly, the data are compatible with (and perhaps even favour) other very different hypotheses about what causes the amount of qualitative research in ECRQ than CBP's allegation of censorship. Figure 2 shows that, rather than the discontinuity we would probably expect from deliberate censorship post 1999, the decline could simply be a trend (albeit a rather noisy one) starting well before the REA and reflecting loss of enthusiasm or funding for qualitative research regardless of ECRQ's actions. CBP do not discuss this point but it seems reasonable that deliberate censorship would produce a sharp break (or at least visibly accelerated decline) in the number of qualitative articles published rather than the noisy long-term trend shown in Figure 2. Interestingly, the data are even compatible with ECRQ protecting qualitative research by publishing anything meeting a minimum standard (that might be below that for quantitative research). If qualitative research happened to be experiencing declining quality control, one would expect to see the observed mode shift over time (from "one qualitative article" to "no qualitative articles" for example). It would be instructive if CBP could identify a statistically meaningful discontinuity in the data or explain how an apparently continuing trend is nonetheless evidence for censorship specifically after 1999. Finally, even after excluding the untypical special issues, it is clear why the results of comparing article counts over short time periods before and after the REA is quite sensitive to the length of these periods. The fraction of qualitative articles is just rather noisy (as one would expect with the small counts involved) and this makes even the simple trend shown in Figure 2 empirically tentative. 
It should also be noted that CBP fail to discuss institutional issues relevant to their hypothesis. Since ECRQ is a good journal with a high submission rate, it is quite likely that articles published in 2000 (and perhaps even in 2001) were actually accepted before the REA. As such, it is not particularly convincing to equate the discontinuity in qualitative publication with the REA year itself. In a similar vein, were a journal disposed to censor qualitative research, it is not clear (and CBP do not make it clear) why it should wait until the REA had become law, rather than starting when it was clear that it would become law or even censoring articles completely independently of the REA.

To sum up, the ways CBP categorise and analyse the articles in ECRQ do not seem (for reasons given) robust enough either qualitatively or quantitatively to serve as evidence for their claim of censorship. There is no unarguable reason why qualitative data analysed quantitatively must be categorised as quantitative rather than qualitative or mixed (and CBP give no indication of how they categorised it at all). CBP's observation of declining qualitative publication in the time period they analyse only seems to hold on the basis of unjustified assumptions but more importantly, even had their categorisation and observations been unquestionable, they offer no evidence at all to link these to one underlying cause rather than another. In fact, while analysing the whole lifetime of ECRQ does appear to show a long-term decline in qualitative publication (which CBP claim for their shorter time periods but do not demonstrate convincingly), this actually makes it harder to reconcile the observed trend with either censorship or indeed any specific change after the REA.

Hopefully no school of qualitative research would take interpretation to mean the arbitrary selection of any hypothesis not flatly contradicted by the data. Rather, it 
would seem to mean narrative (or other) reasons for believing one hypothesis rather than another (and an agnostic view clearly stating the alternatives if there is not a reasonable balance of evidence). Occam's razor also seems relevant here. Even if it were the case that CBP had shown that the rejection of ACS by ECRQ was inappropriate (and I argue throughout this article that they offer no convincing evidence for this), it is still more economical (and thus convincing) to see this rejection as arising from normal failings of peer review than to postulate covert censorship (for which no effective evidence can be offered) to explain the same outcome.

Thus (in a nutshell) CBP's analysis neither seems to follow statistical methodology (with adequate sample sizes and appropriately justified data presentation) nor to follow qualitative practice (involving details of exactly how the research was done and sensitivity to the implications of alternative interpretations in reaching conclusions).

\section{Issues of Interpretation}

Having been fairly well immersed in the style of ECRQ articles through trying to allocate the entire run of the journal to categories by research method, it gives one a curious feeling that CBP present themselves as surprised and upset by the rejection of ACS because it is pretty clear why they got the reviews they did. (These were, if not exactly unexceptionable, then clearly congruent with the ECRQ style.) CBP seem not to have followed advice they cite with approval in their conclusion (p. 684) to "do your homework" on journal policies before submission. As already shown, ECRQ has always been a strongly quantitative journal. Thus CBP's comment that ECRQ “has always published more quantitative than qualitative studies" (p. 682) while strictly true seems like an unjustifiably large understatement. (Compare the boast "I kissed all the 
girls at the party" when, in fact, all those attending were men.) In fact, ECRQ has never published more than a few articles in the style that CBP seem to approve as qualitative. Most of its articles collecting qualitative data actually code that data systematically (with considerable attention to inter-coder reliability and protocols for coding consistency) and analyse it quantitatively and thus might well be regarded as quantitative in the qualitative research community one assumes CBP belong to. Under these circumstances it was perfectly reasonable (and CBP should have foreseen this) for typical reviewers (even those who might call themselves qualitative according to the practices of ECRQ) to ask about inter-coder reliability and systematic coding. Further, CBP really should not assume (again no argument for this interpretation is offered) that asking these questions is a misunderstanding of their own style of qualitative research. (The reviewers may be guilty of no more than the same weakness which CBP display, namely assuming that the way they happen to do qualitative research is - or even should be - the way it is done.) It is also simply not the case, as CBP imply, that only quantitative researchers are concerned about improving the rigour of qualitative research by attention to these matters (Cooper, 2011; Northcote, 2012; Seale, 1999). Despite strongly endorsing the distinctive contribution of qualitative methods, it would concern me considerably if someone else were able to code my data in a very different but still plausible way. There are reasons, even within CBP-style qualitative research, for worrying about reliability and, as I have argued in detail, the arguments and evidence offered by CBP would not soothe the worries of a sceptical reader on this score. It should be said (merely in my opinion) that the review segments quoted by CBP do not suggest misunderstanding but clearly expressed and reasonable concerns about qualitative research rigour. (I have shown in this article how exactly the same issues 
undermine CBP's conclusions. It is not unreasonable that ACS may have suffered the same problems.) The claim that the reviewers are "clearly unfamiliar with qualitative methods" (p. 682) is not justified. (It would be interesting to see if CBP could support this interpretation by detailed analysis of reviewer comments that did not depend on normative views about how qualitative research ought to be done.) The fact that one can reasonably see the quoted evidence so differently from CBP illustrates precisely the concern about the subjective nature of coding raised by the ECRQ reviewers. To reiterate, the argument is not that my interpretation is right but that it is no more obviously wrong than that offered by CBP and thus that some more systematic evaluation based on qualitative or quantitative analysis is therefore necessary before CBP's censorship claim is convincingly supported.

Interestingly, in the light of the content analysis categorising ECRQ articles according to their research methods, CBP's discussion of the reviewing process suggests not censorship but normal administration. Given the dominant qualitative research style in ECRQ (systematic coding for statistical analysis or rigorous qualitative analysis) and the small number of CBP-style qualitative articles published, it is not surprising (as CBP report comments by the editor, pp. 682-683) that ECRQ, at least at that time, did not classify reviewers as being "qualitative" in the sense that CBP wish the term to be used. The editor may reasonably have thought that $\mathrm{s} / \mathrm{he}$ was sending ACS to qualitative reviewers in the sense apparently typical back then at ECRQ. It is clear that (having written to CBP to say that the resubmission would be reviewed differently) the editor does appear to have sent it back to the original reviewers (CBP support this claim by analysis) but this does not seem to me to be evidence of censorship and CBP do not make a case for that. Again, there are at least two equally plausible non- 
conspiracy interpretations of the reported events. The first is that the editor simply made a mistake and forgot to get the article reviewed differently as s/he may genuinely have intended. The second is that the editor actually disagreed with the authors (and shared the views of the reviewers) and, rather than get involved in a did/did not/did too disagreement with CBP, simply gave the decision back to the reviewers. It is actually a journal editor's prerogative (and perhaps even purpose) not to take the same view of an article as the authors and to form their own judgement on the relative strength of author and reviewer arguments. Based on the evidence $\mathrm{CBP}$ present, concerns raised by the reviewers do not seem to me to be obviously inappropriate though CBP have not allowed their readers to see the article the reviewers saw (or even easily access the published version). The fact that such reasonable differences of interpretation exist suggests that CBP need to do proper justificatory work before their claims are even supported, let alone proven. It is not unreasonable, using CBP as a sample of their work, to suppose that the same lack of careful justification was present in the article that ECRQ rejected and CBP claim was censored.

\section{A Different Kind of Publication and Why It May Be Necessary}

Academics may be so used to the usual modes of publication that they give little thought to their underlying logic. It is fairly common for authors to take objection to the work of other authors at article length (Chattoe \& Hamill, 2005; Coles, 2001). At the opposite extreme, some journals publish short comments or corrections regarding relatively factual or technical matters (Gardner, Alpedrinha, \& West, 2012). However, both kinds of responses seem to depend upon a peer review process that effectively selects basically worthwhile articles in the first place. In the first example, the original article has enough merit to deserve an equally lengthy response. In the second example, 
the article is valuable but for some relatively small mistake or lacuna which can easily be addressed in a short note. But what should happen when an article published in a peer-reviewed journal is shown on closer inspection to be very seriously flawed? In the physical sciences, the institution of withdrawing or retracting articles (Van Noorden, 2011) has become more widespread (though often for cases of outright fraud.) Unfortunately, this approach seems to be rare in the social sciences and, in any event, relies heavily on the discretion of editors who may face a conflict of interest in making it obvious that their journal has published something it shouldn't have. One dismissal of this problem might be to admit that peer review is fallible but argue that flawed articles are weeded out by simply not being cited. It should be noted that the logic of this argument might imply that we should check how widely and wisely articles are cited when considering whether to cite them ourselves but I have never heard of anyone doing this. My own anecdotal experience is that I frequently find the arguments of my articles unrecognisable in their citations. Again, however, this comforting folk response relies on tacit assumptions bearing closer scrutiny. If flawed articles cite other flawed articles, it becomes much less clear that quality control will win out post publication. That would depend on how effective peer review turns out to be (and perhaps on other factors like pressure to publish).

This article (and the approach it takes) was thus born out of an anomaly that does not appear to have received discussion in social science. It does not seem that CBP (an article consisting almost entirely of an unsupported but rather serious claim) should have made it through peer review in the first place. However, given that it did, how should the academic community best respond? Reflecting more carefully on the process of review and citation, it seems unwise merely to hope that CBP will be ignored 
(particularly since it makes an unjustified accusation against ECRQ which may affect submissions of qualitative research to that journal and thus have real effects.) My argument is that it is necessary to show exactly why CBP completely fails to support its arguments (in detail and leaving no reasonable room for doubt) so that it is properly recorded in the literature as an article that should not have been published (rather than one with minor errors, one that nonetheless has value through being constructively disagreed with or one that a reader might endorse or disapprove depending on their personal tastes or the tastes in their academic community of practice.)

This logic has also determined the critical approach of the article, which may seem both pedantic and harsh by the standards prevailing in other styles of academic publication. To make an argument that the article should not have been published, it is not sufficient just to sketch some limitations of CBP. It is necessary to demonstrate that the limitations completely undermine the conclusions of the article. It might be argued that CBP should take little responsibility and that it is actually the job of the reviewers and editors is to prevent such articles being published. However, their deliberations are almost entirely inscrutable in individual cases and the academic community still faces the problem of correctly evaluating published research. As such, exhaustive criticism of CBP may seem unkind but appears to be the only available scientific response to the situation that has arisen that can be made properly evidence-based. We cannot know what the editor and reviewers of QI did but we can see what the failings of the result were.

Further, to justify publishing such harsh criticism, the limitations of CBP cannot simply be a matter of personal opinion or of the taste of some particular group (like ECRQ readers). They must clearly arise directly from the content of CBP itself. For this 
reason, my rebuttal focused almost entirely on critical analysis of CBP and made limited use of the extensive literature on qualitative methodology and best practice. This literature may be valuable in helping authors and reviewers before publication (shaping what authors submit and how reviewers can bring pressure to bear in maintaining standards). Unfortunately, CBP shows it is possible to publish an article completely without regard to that literature and it is important for my core claim (that CBP should not have been published) that my argument is not seen as depending on a particular view of qualitative research with which CBP might simply choose to disagree. The intention was therefore to show that CBP fail to support their argument not by the standards of any particular style of qualitative research, but by any standards at all.

Given that this issue has not, to my knowledge, been articulated and thus that this style of article has no precedents, it is also useful to reflect on the wider logic of this situation. My proposed solution is not the only one. For example, some journals like PLOS ONE are now starting to publish peer commentary and allow author responses and reader evaluations of reviews to be part of what is published. However, it seems important for scientific quality that mechanisms for dealing with reviewing failures should be discussed and agreed upon in social science much more widely than they are. This article is thus an attempt to provide both an initial exemplar and a commentary on the wider issues that arise from the publication of CBP and the formulation of an effective response to that. As part of this argument, it is very important to recognise the consequences had the present article not been published (or had not been considered worth writing), namely that the claims of CBP would stand and (more importantly) be endorsed by peer reviewed publication. 
In fact, the quality of evidence and argument in CBP suggest an alternative hypothesis to the claim that ACS was censored. CBP refer to qualitative research being "aced out", American English slang for being cheated or defrauded out of something deserved (in this case publication). Instead, the publication of CBP seems far more like an "own goal", British English slang for an action that harms your team more than the opposition. This is true in two senses.

Firstly, based on the evidence offered here, CBP is exactly the kind of qualitative research that damages the credibility of the method generally because (as quantitative researchers often object), it delivers no robust knowledge (not just by the quantitative standards that CBP disparage but by any standards) and (when subjected to critical analysis) is hard to distinguish evidentially from personal opinion. If the authors want to demonstrate (rather than merely assert) the value of qualitative research, $\mathrm{CBP}$ seems ill designed for that task.

Secondly, in the event that QI readers are persuaded by CBP that censorship is occurring (when there is actually no robust evidence offered that it is), the authors may have caused exactly the harm they presumably wished to avoid, namely that even less qualitative research is published in ECRQ and other top journals in future (Merton 1948). If potential authors believe that the merits of their work are irrelevant to acceptance in a particular journal, they will presumably not submit to that journal.

The final element in my analysis of alleged censorship and the quality of qualitative research involves what happened when I tried to submit my analysis of CBP to QI. QI's reasons for rejection (presented and analysed in the final section) seem to have nothing to do with whether my claims about CBP are correct or not. The successful publication of CBP in QI is thus an own goal for qualitative research in a 
third sense, not only because that article serves as further evidence for the critical view that some qualitative research fails to generate robust knowledge and because (if mistakenly believed) it may discourage future qualitative submissions to ECRQ but also because QI's reaction to my critique raises concerns about whether at least one qualitative research journal evaluates itself impartially and engages scientifically with criticism. This situation obviously has wider implications for academic quality.

Under these circumstances letting CBP's arguments stand and failing to report the QI reviewing process would be scientifically irresponsible of me. Research like CBP cannot safely be ignored (especially if published in peer-reviewed journals.)

\section{The Three Hundred and Sixty Degree Challenge of Qualitative Quality}

By now, the reader may have branded me as just another politically motivated disparager of qualitative research even though this article provides (as CBP does not) evidence and arguments for all its claims. In fact, my career has involved the publication, reviewing, and supervision of qualitative research, and I strongly support CBP's view that qualitative research should be taken seriously and has contributions to make that other research methods cannot. This is precisely why I think articles like CBP should not be allowed to stand. However, what lessons should we draw from the publication of CBP to avoid future own goals in promoting the distinctive value of qualitative research?

The first is that reviewing must clearly be taken much more seriously and, in particular, that this must include agreed strategies for dealing with (inevitable) peer review failure post publication. To protect the credibility of qualitative research, we must not only make sure that we publish the best work we can, but make sure that others do not publish what will bring the approach into disrepute. In the event that such 
research is published, it must be feasible to do something effective and conclusive about it (as discussed in the previous section). We may actually do more good in this regard by providing a rigorous peer review (or critical demolition) than by publishing an article of our own even though academic reward systems increasingly favour publication over systemic maintenance of quality. For example, I have been told that as regards the UK Research Excellence Framework, the present article isn't research despite involving more thorough critical analysis than many articles I have published that were. One risk is that it is currently simply too much effort for too little credit to do what is necessary to enforce academic quality post publication. We cannot necessarily overturn counterproductive bureaucratic systems (that merely count rather than weigh academic output) but we can at least resist their most harmful effects by thinking carefully about the balance of time spent in producing our own work and maintaining the quality of work in others. It may be, too, that my solution to the publication of CBP is generally unfeasible but the severity of the problem that this case study highlights should perhaps focus more attention on other (perhaps more efficient or sustainable) institutional solutions like withdrawing articles or wider use of open peer review.

The second is that a distinctive oddity of CBP is that it does not mention the actual results of ACS. Even the positive review cited on page 683 says the article "takes us into a new world of the child" without saying exactly what has been discovered. If we sincerely believe in the value of qualitative research, it should be possible to make that argument directly by successfully publishing substantive findings in top-quality journals rather than attributing failure to a political conspiracy for which no evidence can be demonstrated. In the same way, if there are legitimate scientific concerns about the use (or overuse) of RCTs in educational research, the best way to promote 
qualitative research would be to show that it can directly challenge their implicit assumptions or findings rather than dismissing the whole approach as CBP try to do. After all, if the idea of RCT as a gold standard is mere rhetoric as CBP claim, surely that obliges them to refute it by something other than more of the same!

The third is that it is very important that we distinguish between genuinely necessary aspects of qualitative methodology (without which it could not deliver what it does) and those that merely make it easier for us to publish mediocre research in communities sharing our prejudices. It is only when research from one qualitative school is exposed to the standards of another - as seen in the response of ECRQ to ACS - that we realise that CBP-style qualitative research rarely provides any explicit justification for the typical absence of coding consistency checks or, in some cases, even systematic coding. It is hard to see how failing to justify this carefully can be necessary to the results of qualitative research and all too easy to see how coder bias or non-systematic coding can simply marginalise data incompatible with a pet theory. In fact, raising the issue of systematic and reproducible coding of narratives is not, as CBP claim, merely a misunderstanding of the true spirit behind the qualitative approach (as if true spirits were a useful concept in social science anyway) but a serious challenge to the quality of any qualitative research which is just as much of a concern to many qualitative researchers themselves (Cooper, 2011; Northcote, 2012; Seale, 1999). If we reject these ideas of systematic and reproducible procedure, we had better have very good reasons, sufficient to convince even our opponents, that we are not just cutting corners. Hopefully this article demonstrates exactly how much can go wrong - and how badly - with the value of qualitative conclusions if procedure is not reproducible and transparent. There is an argument that we should, as long as they are fair minded and 
competent (a qualification which CBP certainly do not disprove for ECRQ reviewers), actually have our articles reviewed by critics rather than supporters. How much better for the credibility of qualitative research and the welfare of society if the value of what we do is apparent even to a statistician or practical politician. The danger of research separating into supposedly non-commensurable communities of practice (which may, in fact, simply not bother to justify their shibboleths) is that they become entirely responsible for their own quality control while there are considerable institutional pressures (like the importance of publication) to weaken this. Once an area is established and has its own journals, associations, and professors, there is a danger that external attempts to impose quality control (like ECRQ's rejection of ACS or my attempt to publish a previous version of my rebuttal of CBP in QI) will be misinterpreted as part of a hostile conspiracy or simply brushed under the carpet. The obvious danger is that quality will then suffer while naïve measures of success (like how much an academic publishes or how many journals exist in a particular field) will be unaffected. On bad days, despite my wholehearted support for the value of qualitative research, it seems that increasing amounts of what is published in this field displays the flaws I have analysed here and, since CBP is an all too typical example, it is instructive both to see exactly how it fails to deliver scientific knowledge and consequently what it reveals about the effectiveness of the peer review process.

\section{The Wider Implications of This Article's Publication Career}

A previous version of this article (available from the author) was submitted to QI in April 2012. It seemed appropriate to give them first refusal on such a severe critique of one of their articles. Initially the article (4 paper copies and an electronic copy for two different recipients) was lost for two months. Then it received an editorial 
rejection on no specific grounds. I queried this. My position was that if my argument was correct, editorial rejection effectively allowed an unsupported claim of censorship by CBP to stand in the literature and, if my argument was wrong, it should be very easy for someone (like the editor or a reviewer) to say why. I was then told by the editor (after a further considerable delay) that the article was rejected because it "used quantitative methodologies to assess a qualitative argument, hence I felt the work did not fit in our journal, which reports qualitative inquiry projects only" (all original correspondence is available from the author). The irony of this reasoning is delicious but the implications for academic quality are disturbing. Firstly, this seems far closer to rejection for methods alone than anything in the treatment of ACS by ECRQ (which received detailed and relevant peer review with its rejection). Secondly, it was possible for QI to reject (without redress) an article calling into question the quality of one of its articles without having to evaluate whether the criticisms involved were actually correct. In particular, the editor of QI appeared not to have noticed that my submission only involved the very same piece of research methodology that they had already published as CBP (namely counting ECRQ articles by research method employed). The only difference is that my article used a graph as a device to show that the raw article counts used by CBP do not properly support their argument. Does this really make my research "quantitative" even though it paid far more attention to issues of interpretation in categorization (even in the use of quantitative data) than CBP did? This would seem a bizarre state of affairs. Thirdly, and most importantly, rejecting my submission without addressing the correctness of its arguments did in fact allow unsupported allegations by CBP to stand in the literature because we currently have no agreed mechanism for marking out articles as reviewing failures independent of editorial discretion. If I am 
right (on the basis of the arguments presented here) and CBP are wrong, then by accepting their article and rejecting mine for no scientific reason, QI is party to misleading its readers about the scholarly value of CBP. Fortunately for scholarship, CBP explicitly accused ECRQ of censorship and thus ECRQ was willing to consider this article. (Nonetheless, to ensure its quality, the current version of this article has undergone 3 significant revisions based on a combination of blind peer review and editorial feedback and I would still be very grateful to have my attention drawn to potential flaws in my argument.) It is very unlikely that any other journal would have been interested in such a specific and detailed analysis (particularly had CBP merely published unsupported claims without specific accusations against another journal). In these circumstances, the maintenance of scientific quality by peer review falls to the ground as journals can effectively prevent what they publish being questioned anywhere where their readers (who are the ones at risk of being influenced by such flawed research) are likely to find it. Any inability to raise serious questions about the quality of research after publication makes it necessary for the quality of published research to depend on infallible reviewing which seems unwise. As it is, most readers of QI probably don't know (and may well never find out) that the CBP article has been refuted here and thus should not be cited. It will be interesting to follow the citation of both articles in the years to come to see whether QI has effectively managed to suppress legitimate and potentially damning criticism by editorial fiat. This suggests a final issue that may impede peer review in best ensuring the quality of published research. The issue is that journal policies may appear administratively innocuous while actually being scientifically harmful. Clearly, QI is a qualitative journal and should not be expected to publish quantitative research routinely. On the other hand, if an article it has 
already published is flawed in ways that can most effectively be shown using quantitative evidence, rejecting it (particularly under editorial discretion alone) has implications that are not purely administrative, namely that the readers of QI will believe the CBP has been deservedly endorsed by publication and that its conclusions still stand. Another example would be a journal policy not to publish short notes. If an article contains a serious flaw that can be briefly explained (like failure to cite relevant research or misquotation), a short note is the most sensible way to point this out both in terms of effort for the critic and impact for the reader. If, as was my experience of the journal Kyklos, the critic is obliged to write an entire article in order to make a short point, the resulting effort is a significant discouragement to communal quality control and there is also a danger that readers will mistake a correction (on a matter of fact) for a debate (in which it is reasonable for people to take sides) and an important academic distinction will be obscured. It may be a matter of opinion whether rational choice is a generally useful theory of human behavior but it is not merely a matter of opinion that the mean is a poor measure of highly skewed data. Treating any criticism of research as equally a matter of opinion largely undermines the scientific approach. The success of academic publishing stands or falls on the weakest link in the process, whether that is the authors, reviewers, or editors. This article has attempted to show in detail why that is the case, why it matters, and why we need to think much more seriously about how we can most effectively deal with reviewing failure after publication. 


\section{References}

Brantlinger, E., Jiminez, R., Klinger, J., Pugach M., \& Richardson, V. (2005).

Qualitative studies in special education. Exceptional Children, 71, 195-207. doi:10.1177/001440290507100205

Ceglowski, D., Bacigalupa, C., \& Peck, E. (2011). Aced out: Censorship of qualitative research in the age of "scientifically based research." Qualitative Inquiry, 17, 679686. doi:10.1177/1077800411415497

Chattoe, E., \& Hamill, H. (2005). It's not who you know - it's what you know about people you don't know that counts: Extending the analysis of crime groups as social networks. British Journal of Criminology, 45, 860-876. doi:10.1093/bjc/azi051

Cole, S., Cole, J., \& Simon, G. (1981). Chance and consensus in peer review. Science, 214, 881-886. doi:10.1126/science.7302566

Coles, N. (2001). It's not what you know - it's who you know that counts: Analysing serious crime groups as social networks. British Journal of Criminology, 41, 580594. doi:10.1093/bjc/41.4.580

Cooper, R. (2011). Appraising qualitative research reports: A developmental approach. The Qualitative Report, 16, 1731-1740. Retrieved from http://www.nova.edu/ssss/QR/QR16-6/cooper.pdf

Gardner, A., Alpedrinha, J., \& West, S. (2012). Correction. The American Naturalist, 179, 554-555. doi:10.1086/664828

Merton, R. (1948). The self-fulfilling prophecy. Antioch Review, 8, 193-210. doi: $10.2307 / 4609267$ 
Northcote, M. (2012). Selecting criteria to evaluate qualitative research. Education Papers and Journal Articles, Paper 38. Retrieved from http://research.avondale.edu.au/edu_papers/38

Peters, D., \& Ceci, S. (1982). Peer-review practices of psychological journals: The fate of published articles, submitted again. Behavioral and Brain Sciences, 5, 187-255. doi:10.1017/S0140525X00011183

Seale, C. (1999). Quality in qualitative research. Qualitative Inquiry, 5, 465-478. doi:10.1177/107780049900500402

Simons, H. (1995). The politics and ethics of educational research in England: Contemporary issues. British Educational Research Journal, 21, 435-450. doi:10.1080/0141192950210401

Van Noorden, R. (2011). Science publishing: The trouble with retractions. Nature, 478, 26-28. doi:10.1038/478026a

Weller, A. C. (2002). Editorial peer review: Its strengths and weaknesses. Medford, NJ: Information Today. 


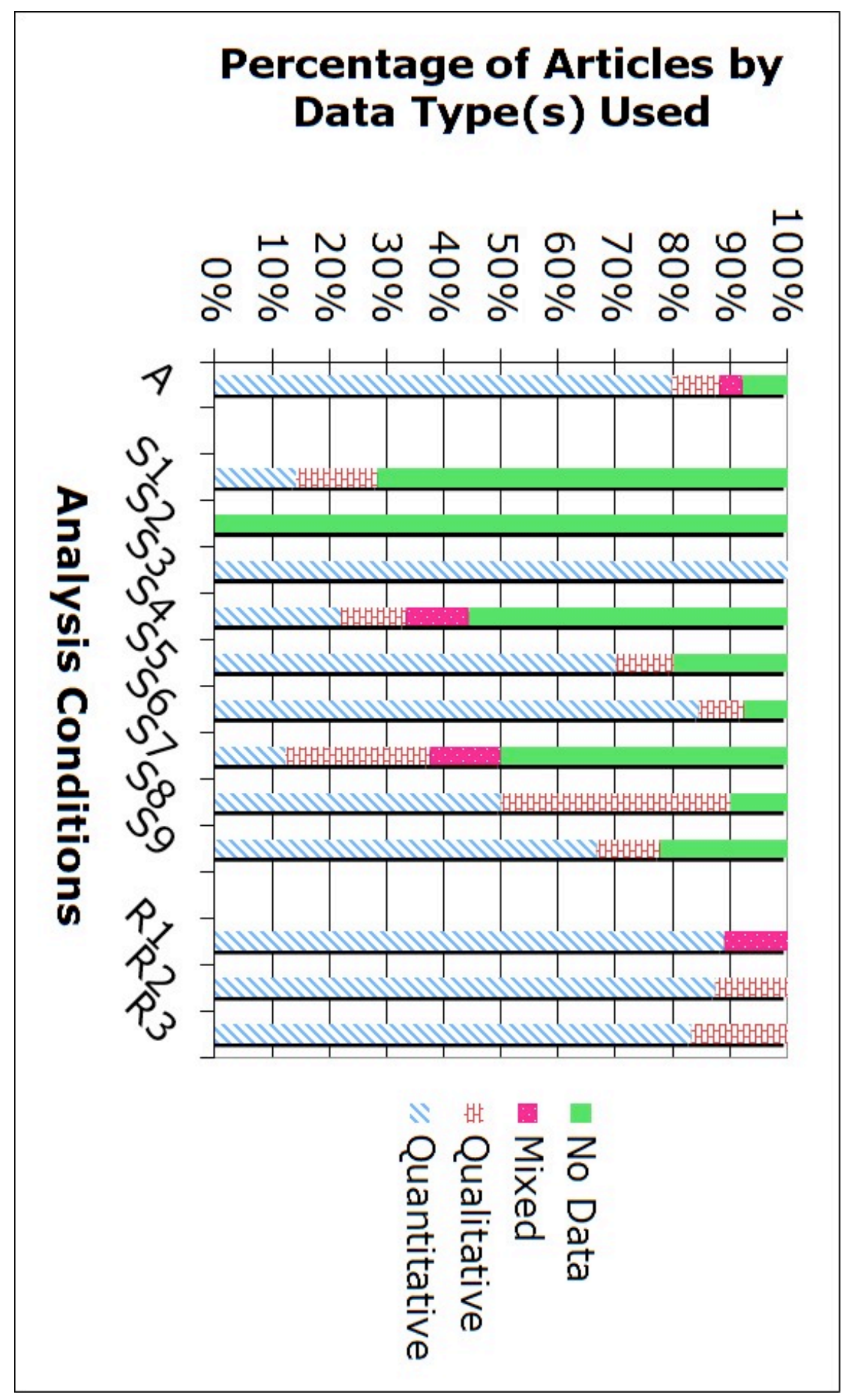

Figure 1. The percentages of ECRQ articles that use various types of data in 13 different conditions. These conditions are: A (Average percentage for non-special issues 1986-2011), S1-S9 (Percentages for all individual special issues 1986-2011), R1-R3 (Percentages for three non-special issues selected randomly 1986-2011). 


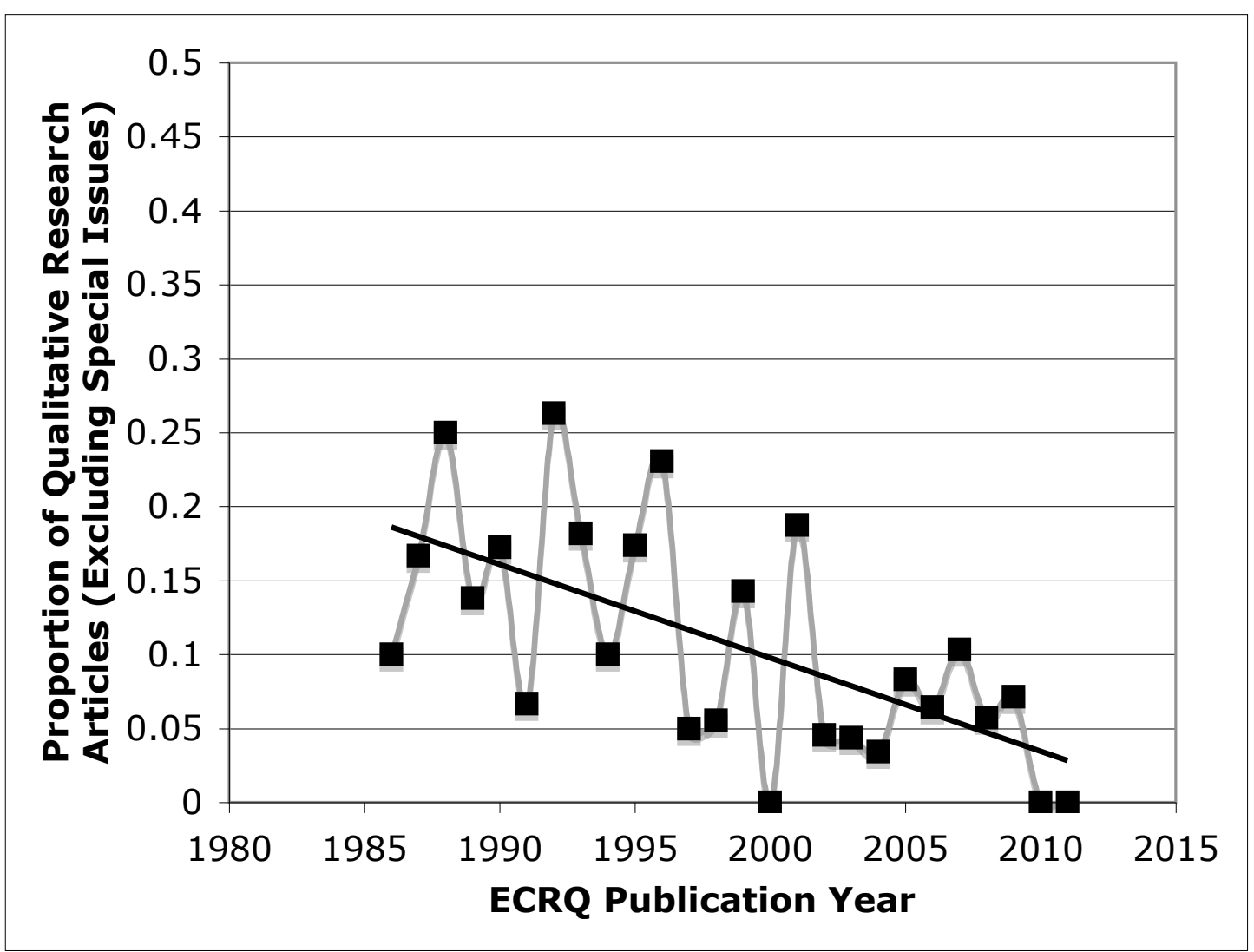

Figure 2. The proportion of qualitative research articles published in ECRQ by year from 1986 to 2011 